\title{
Association of gene polymorphisms with chronic kidney disease in Japanese individuals
}

\author{
TETSURO YOSHIDA ${ }^{1}$, KIMIHIKO KATO ${ }^{2}$, KIYOSHI YOKOI ${ }^{2}$, MITSUTOSHI OGURI $^{3}$, \\ SACHIRO WATANABE ${ }^{4}$, NORIFUMI METOKI ${ }^{5}$, HIDEMI YOSHIDA ${ }^{6}$, KEI SATOH $^{6}$, \\ YUKITOSHI AOYAGI ${ }^{7}$, YOSHINORI NOZAWA ${ }^{8}$ and YOSHIJI YAMADA ${ }^{9}$ \\ ${ }^{1}$ Department of Cardiovascular Medicine, Inabe General Hospital, Inabe; ${ }^{2}$ Department of \\ Cardiovascular Medicine, Gifu Prefectural Tajimi Hospital, Tajimi; ${ }^{3}$ Department of Cardiology, \\ Japanese Red Cross Nagoya First Hospital, Nagoya; ${ }^{4}$ Department of Cardiology, \\ Gifu Prefectural General Medical Center, Gifu; ${ }^{5}$ Department of Internal Medicine, \\ Hirosaki Stroke Center, Hirosaki; ${ }^{6}$ Department of Vascular Biology, Institute of Brain Science, \\ Hirosaki University Graduate School of Medicine, Hirosaki; ${ }^{7}$ Department of Genomics for \\ Longevity and Health, Tokyo Metropolitan Institute of Gerontology, Tokyo; \\ ${ }^{8}$ Gifu International Institute of Biotechnology and Tokai Gakuin University, Kakamigahara; \\ ${ }^{9}$ Department of Human Functional Genomics, Life Science Research Center, Mie University, Tsu, Japan
}

Received April 27, 2009; Accepted June 17, 2009

DOI: 10.3892/ijmm_00000263

\begin{abstract}
Chronic kidney disease (CKD) is recognized as a risk factor not only for end-stage renal disease but also for cardiovascular disease. Early detection and treatment of CKD is a likely key factor for prevention of its complications. Although genetic linkage analyses and association studies have implicated several loci and candidate genes in predisposition to $\mathrm{CKD}$, the genes that underlie genetic susceptibility to this condition have remained largely unknown. The purpose of the present study was to identify genetic variants that confer susceptibility to CKD in Japanese individuals. The study population comprised 4,829 Japanese individuals (2,697 men, 2,132 women), including 757 subjects with CKD [464 men, 293 women; estimated glomerular filtration rate (eGFR) $\left.<50 \mathrm{ml} \mathrm{min} 1.73 \mathrm{~m}^{-2}\right]$ and 4,072 controls $(2,233$ men, 1,839 women; eGFR $\geq 60 \mathrm{ml} \mathrm{min}$ $\left.1.73 \mathrm{~m}^{-2}\right)$. The genotypes for 40 polymorphisms of 39 candidate genes were determined. The chi-square test, multivariable logistic regression analysis with adjustment for covariates, as well as a stepwise forward selection procedure revealed that six polymorphisms of $F 10$, PITRM1, PCSK2,
\end{abstract}

Correspondence to: Dr Yoshiji Yamada, Department of Human Functional Genomics, Life Science Research Center, Mie University, 1577 Kurima-machiya, Tsu, Mie 514-8507, Japan E-mail: yamada@gene.mie-u.ac.jp

Key words: genetics, polymorphism, chronic kidney disease, endstage renal disease
$J P H 3, M Y O 7 B$, and $A K A P 12$ were related $(\mathrm{P}<0.05)$ to the prevalence of CKD. Among these polymorphisms, the $\mathrm{C} \rightarrow \mathrm{T}$ polymorphism of F10 (rs5962) was most significantly associated with this condition. Determination of genotypes for the $\mathrm{C} \rightarrow \mathrm{T}$ polymorphism of $\mathrm{F} 10$ may prove informative for assessment of genetic risk for CKD in Japanese individuals.

\section{Introduction}

A current epidemic of chronic kidney disease (CKD) is a major health problem worldwide. In Japan, the number of new patients with end-stage renal disease (ESRD) has been increasing during the last four decades (1). Individuals with CKD are at increased risk not only for ESRD but also for a poor cardiovascular outcome and premature death $(2,3)$. Disease prevention is an important strategy for reducing the overall burden of CKD and ESRD, and the identification of markers for disease risk is essential both for risk prediction and for potential intervention to reduce the chance of future cardiovascular events (4).

Although genetic linkage analyses (5) and association studies (6-10) have implicated several loci and candidate genes in predisposition to CKD, the genes that confer susceptibility to this condition remain to be identified definitively. In addition, given the ethnic differences in lifestyle and environmental factors as well as in genetic background and renal function, it is important to examine genetic variants related to CKD in each ethnic group. We have now performed an association study for 40 polymorphisms of 39 candidate genes and CKD in 4,829 Japanese individuals. The purpose of the present study was to identify genetic variants that confer susceptibility to CKD and thereby to provide a basis for the personalized prevention of this condition. 
Table I. The 40 polymorphisms examined in the present study.

\begin{tabular}{|c|c|c|c|c|}
\hline Locus & Gene & Symbol & SNP & dbSNP \\
\hline $1 \mathrm{p} 36.31-\mathrm{p} 36.11$ & Acyl-CoA thioesterase 7 & ACOT7 & $\mathrm{A} \rightarrow \mathrm{C}$ & rs3789485 \\
\hline $2 \mathrm{p} 25.3$ & Collectin sub-family member 11 & COLEC11 & $\mathrm{C} \rightarrow \mathrm{T}$ & rs6739899 \\
\hline $2 \mathrm{q} 21.1$ & Myosin VIIB & MYO7B & $\mathrm{C} \rightarrow \mathrm{T}$ & rs 13015157 \\
\hline $2 q 25$ & Peroxidasin homolog (Drosophila) & $P X D N$ & $A \rightarrow G$ & rs12617409 \\
\hline $4 \mathrm{p} 16.3$ & Phosphatidylinositol glycan anchor biosynthesis, class G & $P I G G$ & $\mathrm{C} \rightarrow \mathrm{T}$ & rs4499656 \\
\hline $4 q 22.1$ & Family with sequence similarity 13 , member A 1 & FAM13A1 & $A \rightarrow G$ & rs6532094 \\
\hline $5 q 35.1-q 35.2$ & Endoplasmic reticulum-golgi intermediate compartment 1 & ERGC1 & $A \rightarrow G$ & rs2339745 \\
\hline $5 q 35.3$ & Collagen, type XXIII, $\alpha 1$ & COL23A1 & $\mathrm{G} \rightarrow \mathrm{T}$ & rs535858 \\
\hline $6 q 24-q 25$ & A kinase (PRKA) anchor protein (gravin) 12 & $A K A P 12$ & $A \rightarrow G$ & rs756009 \\
\hline $6 q 25.3$ & Poly (A)-specific ribonuclease (PARN)-like domain containing 1 & $P N L D C 1$ & $\mathrm{C} \rightarrow \mathrm{G}$ & rs13209678 \\
\hline $7 \mathrm{p} 36$ & Protein tyrosine phosphatase, receptor type, N polypeptide 2 & PTPRN2 & $\mathrm{C} \rightarrow \mathrm{T}$ & rs 1638021 \\
\hline $8 \mathrm{q} 24.3$ & Trafficking protein particle complex 9 & TRAPPC9 & $\mathrm{C} \rightarrow \mathrm{T}$ & rs12679196 \\
\hline 9 p13.3 & Receptor tyrosine kinase-like orphan receptor 2 & ROR2 & $A \rightarrow G$ & rs10992119 \\
\hline $9 q 22.3$ & WNK lysine deficient protein kinase 2 & $W N K 2$ & $\mathrm{G} \rightarrow \mathrm{T}$ & rs16936752 \\
\hline $9 q 32$ & Zinc finger protein 618 & ZNF618 & $\mathrm{C} \rightarrow \mathrm{T}$ & rs1330171 \\
\hline $10 \mathrm{p} 15.3$ & WD repeat domain 37 & WDR37 & $A \rightarrow G$ & rs6560711 \\
\hline $10 \mathrm{p} 15.2$ & Pitrilysin metallopeptidase 1 & PITRM1 & $A \rightarrow G$ & rs7094698 \\
\hline $10 \mathrm{p} 15.2$ & Pitrilysin metallopeptidase 1 & PITRM1 & $\mathrm{C} \rightarrow \mathrm{G}$ & rs7898290 \\
\hline $10 \mathrm{q} 11.2$ & Arachidonate 5-lipoxygenase & ALOX5 & $A \rightarrow G$ & rs7913948 \\
\hline $10 q 11.23$ & Oxoglutarate dehydrogenase-like & $O G D H L$ & $A \rightarrow G$ & rs11101224 \\
\hline $11 \mathrm{p} 11.2$ & Glycosyltransferase-like 1B & GYLTL1B & $\mathrm{C} \rightarrow \mathrm{G}$ & rs10838532 \\
\hline $11 \mathrm{q} 13.4$ & NAD synthetase 1 & NADSYN1 & $\mathrm{A} \rightarrow \mathrm{C}$ & rs3814731 \\
\hline $13 q 12.2$ & Polymerase (RNA) I polypeptide D, $16 \mathrm{k} \mathrm{Da}$ & POLRID & $\mathrm{C} \rightarrow \mathrm{T}$ & rs14105 \\
\hline $13 q 34$ & Coagulation factor $\mathrm{X}$ & $F 10$ & $\mathrm{C} \rightarrow \mathrm{T}$ & rs5962 \\
\hline $14 \mathrm{p} 31$ & Inositol 1,3,4-triphosphate 5/6 kinase & ITPKI & $\mathrm{C} \rightarrow \mathrm{T}$ & rs2295394 \\
\hline $14 q 22.1$ & Atlastin GTPase 1 & ATL1 & $\mathrm{C} \rightarrow \mathrm{T}$ & rs4901043 \\
\hline $16 \mathrm{p} 13.3$ & Transducin (ß)-like 3 & $T B L 3$ & $\mathrm{~A} \rightarrow \mathrm{C}$ & rs8053843 \\
\hline $16 \mathrm{q} 23.2$ & Chromodomain protein, Y-like 2 & $C D Y L 2$ & $A \rightarrow G$ & rs 17823453 \\
\hline $16 q 24$ & COX4 neighbor & $C O X 4 N B$ & $\mathrm{C} \rightarrow \mathrm{T}$ & rs301164 \\
\hline $16 \mathrm{q} 24.3$ & Junctophilin 3 & $J P H 3$ & $\mathrm{C} \rightarrow \mathrm{G}$ & rs2562059 \\
\hline $17 \mathrm{q} 21$ & Wingless-type MMTV integration site family, member 3 & WNT3 & $\mathrm{C} \rightarrow \mathrm{G}$ & rs 199515 \\
\hline $17 \mathrm{q} 23.2$ & Membrane-associated ring finger $(\mathrm{C} 3 \mathrm{HC} 4) 10$ & MARCH1O & $\mathrm{C} \rightarrow \mathrm{T}$ & rs2251393 \\
\hline $17 q 25.3$ & Tubulin folding cofactor D & $T B C D$ & $\mathrm{~A} \rightarrow \mathrm{C}$ & rs8076476 \\
\hline $18 \mathrm{q} 11.2$ & Laminin, $\alpha 3$ & LAMA3 & $\mathrm{G} \rightarrow \mathrm{T}$ & rs12373237 \\
\hline 20p11.23-p11.21 & Acyl-CoA synthetase short-chain family member 1 & ACSS1 & $\mathrm{C} \rightarrow \mathrm{T}$ & rs6138473 \\
\hline 20p11.2 & Proprotein convertase subtilisin/kexin type 2 & PCSK2 & $\mathrm{C} \rightarrow \mathrm{G}$ & rs6080699 \\
\hline $20 \mathrm{q} 13.3$ & Cadherin 4 , type 1 , R-cadherin (retinal) & $\mathrm{CDH} 4$ & $A \rightarrow G$ & rs6142884 \\
\hline $20 q 13.3$ & Synovial sarcoma translocation gene on chromosome 18 -like 1 & SS18L1 & $\mathrm{C} \rightarrow \mathrm{T}$ & rs2427254 \\
\hline $21 \mathrm{q} 22.3$ & Trefoil factor 1 & $T F F 1$ & $\mathrm{C} \rightarrow \mathrm{G}$ & rs13051704 \\
\hline $22 \mathrm{q} 11.21$ & Armadillo repeat gene deletes in velocardiofacial syndrome & $A R V C F$ & $A \rightarrow G$ & rs2073746 \\
\hline
\end{tabular}

\section{Materials and methods}

Study population. The study population comprised 4,829 unrelated Japanese individuals (2,697 men, 2,132 women) who either visited outpatient clinics of or were admitted to one of the participating hospitals (Gifu Prefectural General Medical Center and Gifu Prefectural Tajimi Hospital in Gifu Prefecture, Japan; and Hirosaki University Hospital, Reimeikyo Rehabilitation Hospital, and Hirosaki Stroke Center in Aomori Prefecture, Japan) between October 2002 and March 2008 because of various symptoms or for an annual health checkup, or who were recruited to a population-based prospective cohort study of aging and age-related diseases in Gunma Prefecture, Japan.
Estimated glomerular filtration rate (eGFR) was calculated with the use of the simplified prediction equation derived from the modified version of that described in the Modification of Diet in Renal Disease (MDRD) Study as proposed by the Japanese Society of Nephrology (11), eGFR $\left(\mathrm{ml} \mathrm{min}^{-1} 1.73 \mathrm{~m}^{-2}\right)=$ $194 x$ [age (years) $]^{-0.287} \mathrm{x}$ [serum creatinine $\left.(\mathrm{mg} / \mathrm{dl})\right]^{-1.094} \mathrm{x}[0.739$ if female]. The National Kidney Foundation-Kidney Disease Outcomes Quality Initiative guidelines recommend a diagnosis of CKD if eGFR is $<60 \mathrm{ml} \mathrm{min} \mathrm{m}^{-1} 1.73 \mathrm{~m}^{-2}$ (4). Nonlinear relations between GFR and the risk of adverse events, such as death, cardiovascular events, and hospitalization, have been demonstrated, with an increased risk being associated with an eGFR of $<60 \mathrm{ml} \mathrm{min}{ }^{-1} 1.73 \mathrm{~m}^{-2}$ and the risk rising further 


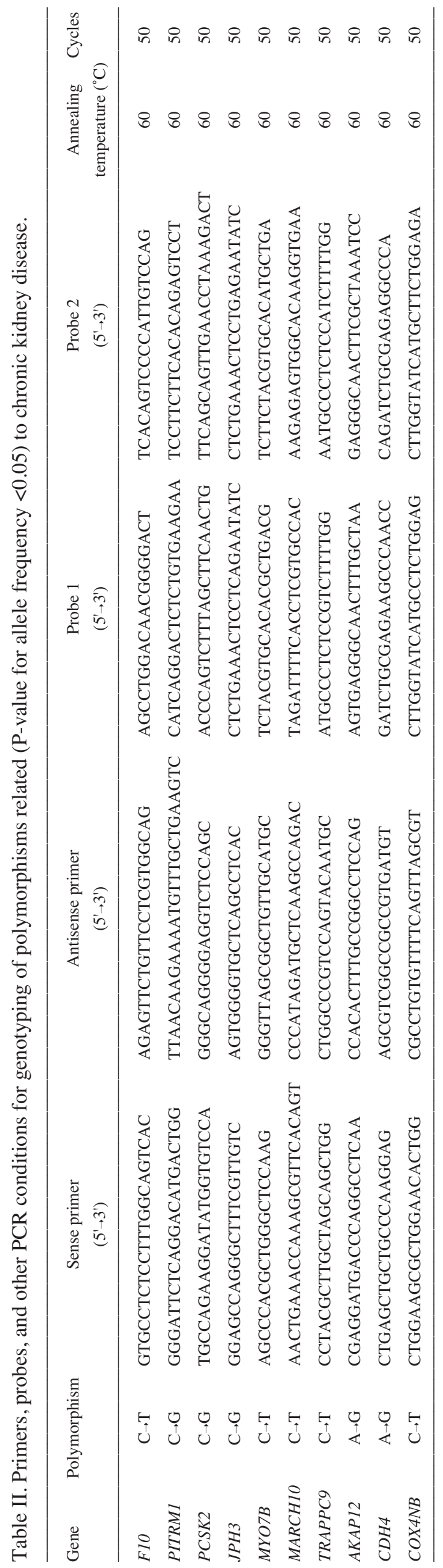


Table III. Characteristics of subjects with chronic kidney disease (CKD) and controls.

\begin{tabular}{|c|c|c|c|}
\hline Characteristic & CKD & Controls & P-value \\
\hline No. of subjects & 757 & 4072 & \\
\hline Age (years) & $71.3 \pm 9.2$ & $65.2 \pm 10.5$ & $<0.0001$ \\
\hline Sex (male/female, \%) & $61.3 / 38.7$ & $54.8 / 45.2$ & 0.0010 \\
\hline Body mass index $\left(\mathrm{kg} / \mathrm{m}^{2}\right)$ & $23.5 \pm 4.6$ & $23.5 \pm 4.6$ & 0.5619 \\
\hline Smoker $(\%)$ & 17.6 & 24.1 & $<0.0001$ \\
\hline Hypertension (\%) & 75.7 & 54.4 & $<0.0001$ \\
\hline Systolic blood pressure $(\mathrm{mmHg})$ & $149 \pm 27$ & $140 \pm 23$ & $<0.0001$ \\
\hline Diastolic blood pressure $(\mathrm{mmHg})$ & $78 \pm 16$ & $78 \pm 14$ & 0.1973 \\
\hline Hypercholesterolemia (\%) & 39.6 & 33.0 & 0.0004 \\
\hline Serum total cholesterol $(\mathrm{mmol} / \mathrm{l})$ & $5.23 \pm 1.07$ & $5.17 \pm 0.99$ & 0.2138 \\
\hline Serum triglyceride $(\mathrm{mmol} / \mathrm{l})$ & $1.76 \pm 1.03$ & $1.59 \pm 1.09$ & $<0.0001$ \\
\hline Serum HDL-cholesterol (mmol/l) & $1.30 \pm 0.42$ & $1.40 \pm 0.39$ & $<0.0001$ \\
\hline Diabetes mellitus (\%) & 43.3 & 26.4 & $<0.0001$ \\
\hline Fasting plasma glucose $(\mathrm{mmol} / \mathrm{l})$ & $7.24 \pm 3.48$ & $6.74 \pm 2.93$ & 0.0002 \\
\hline Blood glycosylated hemoglobin (\%) & $6.24 \pm 1.66$ & $6.05 \pm 8.92$ & $<0.0001$ \\
\hline Serum creatinine $(\mu \mathrm{mol} / \mathrm{l})$ & $149.9 \pm 169.7$ & $61.7 \pm 12.6$ & $<0.0001$ \\
\hline eGFR $\left(\mathrm{ml} \mathrm{min}{ }^{-1} 1.73 \mathrm{~m}^{-2}\right)$ & $39.3 \pm 11.1$ & $79.3 \pm 16.9$ & $<0.0001$ \\
\hline
\end{tabular}

Quantitative data are means \pm SD. HDL, high density lipoprotein; eGFR, estimated glomerular filtration rate.

markedly when values fall $<45 \mathrm{ml} \mathrm{min}^{-1} 1.73 \mathrm{~m}^{-2}$ (12). In addition, the rate of GFR decline was significantly higher in Japanese individuals younger than 70 years with an initial value of $<50 \mathrm{ml} \mathrm{min}-11.73 \mathrm{~m}^{-2}$, suggestive of a poor outcome for such individuals (13). We thus adopted the criterion of an eGFR of $<50 \mathrm{ml} \mathrm{min}^{-1} 1.73 \mathrm{~m}^{-2}$ for diagnosis of CKD in the present study. On the basis of this criterion, 757 subjects (464 men, 293 women) were diagnosed with CKD. The control subjects comprised 4,072 individuals (2,233 men, 1,839 women) whose eGFR was $\geq 60 \mathrm{ml} \mathrm{min}{ }^{-1} 1.73 \mathrm{~m}^{-2}$. The control subjects were recruited from community-dwelling healthy individuals or the patients who visited outpatient clinics regularly for treatment of various common diseases. Subjects with CKD and controls thus either had or did not have conventional risk factors for CKD, including hypertension (systolic blood pressure of $\geq 140 \mathrm{mmHg}$ or diastolic blood pressure of $\geq 90 \mathrm{mmHg}$, or both, or taking antihypertensive medication), diabetes mellitus (fasting blood glucose of $\geq 6.93 \mathrm{mmol} / \mathrm{l}$ or hemoglobin $\mathrm{A}_{1 \mathrm{c}}$ of $\geq 6.5 \%$, or both, or taking antidiabetes medication), or hypercholesterolemia (serum total cholesterol of $\geq 5.72 \mathrm{mmol} / \mathrm{l}$ or taking lipid-lowering medication). The study protocol complied with the Declaration of Helsinki and was approved by the Committees on the Ethics of Human Research of Mie University Graduate School of Medicine, Hirosaki University Graduate School of Medicine, Gifu International Institute of Biotechnology, Tokyo Metropolitan Institute of Gerontology, and participating hospitals. Written informed consent was obtained from each participant.

Selection of polymorphisms. Our aim was to identify genetic variants associated with CKD in the Japanese population in a case-control association study by examining the relations of candidate gene polymorphisms to this condition. Polymorphisms examined in the present study (Table I) were selected from genome-wide association studies of ischemic stroke (14) and myocardial infarction. Given that atherosclerosis is an important risk factor for CKD (15), genetic variants related to atherosclerotic disease such as ischemic stroke and myocardial infarction might also contribute to the development of CKD. We have not examined the relation of these polymorphisms in the previous studies $(7,8,16-19)$.

Genotyping of polymorphisms. Venous blood $(7 \mathrm{ml})$ was collected into tubes containing $50 \mathrm{mmol} / \mathrm{l}$ ethylenediaminetetraacetic acid (disodium salt), and genomic DNA was isolated with a kit (Genomix; Talent, Trieste, Italy). Genotypes of the 40 polymorphisms were determined at G\&G Science (Fukushima, Japan) by a method that combines the polymerase chain reaction (PCR) and sequence-specific oligonucleotide probes with suspension array technology (Luminex, Austin, TX). Primers, probes, and other PCR conditions for genotyping polymorphisms found to be related (P-value for allele frequency of $<0.05$ ) to CKD by the chi-square test are shown in Table II. Detailed genotyping methodology was described previously (20).

Statistical analysis. Quantitative data were compared between subjects with CKD and controls by the unpaired Student's t test. Categorical data were compared by the chi-square test. Allele frequencies were estimated by the gene counting method, and the chi-square test was used to identify departures from Hardy-Weinberg equilibrium. In the initial screen, the allele frequencies of each polymorphism were compared between subjects with CKD and controls by the chi-square test. Given the multiple comparisons of genotypes with CKD, the false discovery rate (FDR) was calculated from the distribution of $\mathrm{P}$ values for allele frequency of the 40 polymorphisms (21). Polymorphisms with a P-value for allele frequency of $<0.05$ 
Table IV. Genotype distributions of polymorphisms related (P-value for allele frequency <0.05) to chronic kidney disease (CKD) as determined by the chi-square test.

\begin{tabular}{|c|c|c|c|c|c|c|}
\hline Gene symbol & SNP & dbSNP & $\mathrm{CKD}^{\mathrm{a}}$ & Controls $^{\mathrm{a}}$ & $\mathrm{P}$-value & FDR \\
\hline \multirow[t]{4}{*}{ F10 } & $\mathrm{C} \rightarrow \mathrm{T}$ & rs5962 & & & 0.0011 & 0.0437 \\
\hline & $\mathrm{CC}$ & & 747 (99.6) & 4037 (99.9) & & \\
\hline & $\mathrm{CT}$ & & $3(0.4)$ & $1(0.1)$ & & \\
\hline & $\mathrm{TT}$ & & $0(0)$ & $0 \quad(0)$ & & \\
\hline \multirow[t]{4}{*}{ PITRM1 } & $\mathrm{C} \rightarrow \mathrm{G}$ & rs7898290 & & & 0.0074 & 0.0815 \\
\hline & $\mathrm{CC}$ & & $298 \quad(5.9)$ & $1430 \quad(6.1)$ & & \\
\hline & $\mathrm{CG}$ & & $352(33.9)$ & $1946(36.7)$ & & \\
\hline & GG & & $100(60.2)$ & $662(57.2)$ & & \\
\hline \multirow[t]{4}{*}{ PCSK2 } & $\mathrm{C} \rightarrow \mathrm{G}$ & rs6080699 & & & 0.0098 & 0.0815 \\
\hline & $\mathrm{CC}$ & & $42 \quad(5.6)$ & $338 \quad(8.4)$ & & \\
\hline & CG & & $292(38.9)$ & $1611(39.9)$ & & \\
\hline & GG & & $416(55.5)$ & $2089(51.7)$ & & \\
\hline \multirow[t]{4}{*}{ JPH3 } & $\mathrm{C} \rightarrow \mathrm{G}$ & rs 2562059 & & & 0.0102 & 0.0815 \\
\hline & $\mathrm{CC}$ & & $539(71.9)$ & $2711(67.2)$ & & \\
\hline & $\mathrm{CG}$ & & $193(25.8)$ & $1202(29.8)$ & & \\
\hline & GG & & $17 \quad(2.3)$ & $121 \quad(3.0)$ & & \\
\hline \multirow{4}{*}{$M Y O 7 B$} & $\mathrm{C} \rightarrow \mathrm{T}$ & rs 13015157 & & & 0.0116 & 0.0815 \\
\hline & $\mathrm{CC}$ & & $569(75.9)$ & $3244(80.3)$ & & \\
\hline & $\mathrm{CT}$ & & $171(22.8)$ & $738(18.3)$ & & \\
\hline & $\mathrm{TT}$ & & $10 \quad(1.3)$ & $56 \quad(1.4)$ & & \\
\hline \multirow[t]{4}{*}{ MARCHIO } & $\mathrm{C} \rightarrow \mathrm{T}$ & rs 2251393 & & & 0.0122 & 0.0815 \\
\hline & $\mathrm{CC}$ & & $17(2.3)$ & $55 \quad(1.4)$ & & \\
\hline & $\mathrm{CT}$ & & $179(23.8)$ & $847(21.0)$ & & \\
\hline & TT & & $555(73.9)$ & $3128(77.6)$ & & \\
\hline \multirow[t]{4}{*}{ TRAPPC9 } & $\mathrm{C} \rightarrow \mathrm{T}$ & rs 12679196 & & & 0.0245 & 0.1380 \\
\hline & $\mathrm{CC}$ & & $507(67.5)$ & $2510(62.3)$ & & \\
\hline & $\mathrm{CT}$ & & 215 (28.6) & $1370(34.0)$ & & \\
\hline & $\mathrm{TT}$ & & $29 \quad(3.9)$ & $150 \quad(3.7)$ & & \\
\hline \multirow[t]{4}{*}{$A K A P 12$} & $\mathrm{~A} \rightarrow \mathrm{G}$ & rs756009 & & & 0.0276 & 0.1380 \\
\hline & $\mathrm{AA}$ & & $0 \quad(0)$ & $1 \quad(0.1)$ & & \\
\hline & $\mathrm{AG}$ & & $7 \quad(0.9)$ & $85 \quad(2.1)$ & & \\
\hline & GG & & $743(99.1)$ & $3952(97.8)$ & & \\
\hline \multirow[t]{4}{*}{$\mathrm{CDH} 4$} & $\mathrm{~A} \rightarrow \mathrm{G}$ & rs6142884 & & & 0.0410 & 0.1821 \\
\hline & $\mathrm{AA}$ & & $17 \quad(2.3)$ & $65 \quad(1.6)$ & & \\
\hline & $\mathrm{AG}$ & & $182(24.3)$ & 878 (21.7) & & \\
\hline & GG & & $551(73.4)$ & 3095 (76.7) & & \\
\hline \multirow[t]{4}{*}{$C O X 4 N B$} & $\mathrm{C} \rightarrow \mathrm{T}$ & rs301164 & & & 0.0457 & 0.0744 \\
\hline & $\mathrm{CC}$ & & $55 \quad(7.3)$ & $227 \quad(5.6)$ & & \\
\hline & $\mathrm{CT}$ & & $286(38.2)$ & $1484(36.8)$ & & \\
\hline & $\mathrm{TT}$ & & $408(54.5)$ & $2323(57.6)$ & & \\
\hline
\end{tabular}

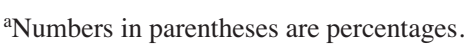

were further examined by multivariable logistic regression analysis with adjustment for covariates. Multivariable logistic regression analysis was thus performed with CKD as a dependent variable and independent variables including age, sex (0, woman; 1 , man), body mass index (BMI), smoking status $(0$, nonsmoker; 1 , smoker $)$, history of hypertension, diabetes mellitus, or hypercholesterolemia ( 0 , no history; 1 , positive history), and genotype of each polymorphism, and the $\mathrm{P}$-value, odds ratio, and $95 \%$ confidence interval were calculated. Each genotype was assessed according to dominant, recessive, and additive genetic models. Additive models included the additive 1 model (heterozygotes versus wild-type homozygotes) and the additive 2 model (variant homozygotes versus wildtype homozygotes), which were analyzed simultaneously with a single statistical model. We also performed a stepwise forward selection procedure to examine the effects of genotypes as well as of other covariates on CKD; each genotype was examined according to a dominant or recessive model on the basis of statistical significance in the multivariable logistic regression analysis. The P-levels for inclusion in and exclusion 
Table V. Hardy-Weinberg P-values in subjects with chronic kidney disease (CKD) and controls

\begin{tabular}{lcccc}
\hline Gene & Polymorphism & dbSNP & CKD & Controls \\
\hline F10 & $\mathrm{C} \rightarrow \mathrm{T}$ & rs5962 & 0.9562 & 0.9937 \\
PITRM1 & $\mathrm{C} \rightarrow \mathrm{G}$ & $\mathrm{rs} 7898290$ & 0.8055 & 0.9991 \\
PCSK2 & $\mathrm{C} \rightarrow \mathrm{G}$ & $\mathrm{rs} 6080699$ & 0.3191 & 0.2717 \\
JPH3 & $\mathrm{C} \rightarrow \mathrm{G}$ & $\mathrm{rs} 2562059$ & 0.9548 & 0.3783 \\
MYO7B & $\mathrm{C} \rightarrow \mathrm{T}$ & $\mathrm{rs} 13015157$ & 0.4778 & 0.0597 \\
MARCH10 & $\mathrm{C} \rightarrow \mathrm{T}$ & $\mathrm{rs} 2251393$ & 0.5695 & 0.7847 \\
TRAPPC9 & $\mathrm{C} \rightarrow \mathrm{T}$ & $\mathrm{rs} 12679196$ & 0.3039 & $\mathbf{0 . 0 2 7 4}$ \\
AKAP12 & $\mathrm{A} \rightarrow \mathrm{G}$ & $\mathrm{rs} 756009$ & 0.8978 & 0.4326 \\
CDH4 & $\mathrm{A} \rightarrow \mathrm{G}$ & $\mathrm{rs} 6142884$ & 0.6680 & 0.7629 \\
COX4NB & $\mathrm{C} \rightarrow \mathrm{T}$ & $\mathrm{rs} 301164$ & 0.6175 & 0.6191 \\
\hline
\end{tabular}

P-values of $<0.05$ are shown in bold.

from the model were 0.25 and 0.1 , respectively. With the exception of the initial screen by the chi-square test (FDR $<0.05$ ), a P-value of $<0.05$ was considered statistically significant. Statistical significance was examined by two-sided tests performed with JMP version 6.0 and JMP Genomics version 3.2 software (SAS Institute, Cary, NC).

\section{Results}

The characteristics of the 4,829 study subjects are shown in Table III. Age, the frequency of male subjects, systolic blood pressure, serum concentrations of triglycerides and creatinine, fasting plasma glucose level, blood glycosylated hemoglobin content, and the prevalence of hypertension, diabetes mellitus, and hypercholesterolemia were greater, whereas serum concentration of high density lipoprotein (HDL)-cholesterol, eGFR, and the percentage of smokers were lower in subjects with CKD than in controls.

Comparison of allele frequencies with the chi-square test revealed that 10 polymorphisms were related ( $\mathrm{P}$-value of $<0.05$ for allele frequency) to the prevalence of CKD (Table IV). Among these polymorphisms, the $\mathrm{C} \rightarrow \mathrm{T}$ polymorphism of $\mathrm{F} 10$ (rs5962) was significantly (FDR <0.05) associated with this condition. The genotype distributions of all 10 polymorphisms, with the exception of the $\mathrm{C} \rightarrow \mathrm{T}$ polymorphism of TRAPPC 9 in control subjects, were in Hardy-Weinberg equilibrium both in individuals with CKD and in controls (Table V); the $\mathrm{C} \rightarrow \mathrm{T}$ polymorphism of TRAPPC 9 was therefore excluded from subsequent analysis.

Multivariable logistic regression analysis with adjustment for age, sex, BMI, smoking status, and the prevalence of hypertension, diabetes mellitus, and hypercholesterolemia revealed that the $\mathrm{C} \rightarrow \mathrm{T}$ polymorphism of $F 10$ (dominant and additive 1 models), the $\mathrm{C} \rightarrow \mathrm{G}$ polymorphism of PITRMI (dominant and additive 2 models), the $\mathrm{C} \rightarrow \mathrm{G}$ polymorphism of PCSK2 (dominant and additive 2 models), the $\mathrm{C} \rightarrow \mathrm{G}$ polymorphism of $\mathrm{JPH} 3$ (dominant and additive 1 models), the $\mathrm{C} \rightarrow \mathrm{T}$ polymorphism of $M Y O 7 B$ (dominant and additive 1 models), the $\mathrm{C} \rightarrow \mathrm{T}$ polymorphisms of $M A R C H 10$ (recessive model), and the $\mathrm{A} \rightarrow \mathrm{G}$ polymorphism of $A K A P 12$ (recessive

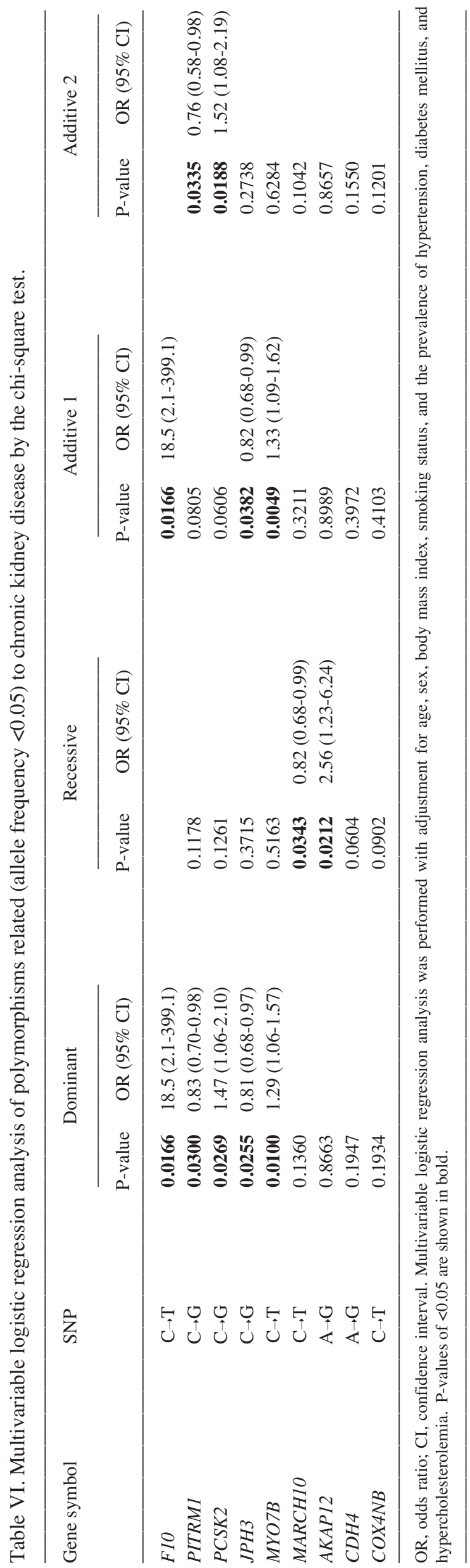


Table VII. Effects of genotypes and other characteristics on chronic kidney disease determined by a stepwise forward selection procedure $(\mathrm{P}<0.05)$.

\begin{tabular}{lcc}
\hline Variable & P-value & $\mathrm{R}^{2}$ \\
\hline Age & $<0.0001$ & 0.0581 \\
Diabetes mellitus & $<0.0001$ & 0.0215 \\
Hypertension & $<0.0001$ & 0.0154 \\
Sex & 0.0002 & 0.0034 \\
Smoking & 0.0031 & 0.0022 \\
F10 $(C T+T T$ versus $C C)$ & 0.0065 & 0.0018 \\
$A K A P 12(G G$ versus $A A+A G)$ & 0.0095 & 0.0016 \\
MYO7B $(C T+T T$ versus $C C)$ & 0.0113 & 0.0016 \\
JPH3 $(C G+G G$ versus $C C)$ & 0.0204 & 0.0014 \\
PCSK2 $(C G+G G$ versus $C C)$ & 0.0254 & 0.0013 \\
PITRM1 $(C G+G G$ versus $C C)$ & 0.0286 & 0.0011 \\
Hypercholesterolemia & 0.0459 & 0.0010 \\
\hline
\end{tabular}

$\mathrm{R}^{2}$, contribution rate.

model) were significantly $(\mathrm{P}<0.05)$ associated with the prevalence of CKD (Table VI). The $T$ allele of F10, G allele of PCSK2, $T$ allele of $M Y O 7 B$, and $G$ allele of $A K A P 12$ were risk factors for CKD, whereas the $G$ allele of PITRM1, $G$ allele of $J P H 3$, and $T$ allele of $M A R C H 1 O$ were protective against this condition.

Finally, we performed a stepwise forward selection procedure to examine the effects of genotypes for the seven polymorphisms associated with CKD by multivariable logistic regression analysis as well as of age, sex, BMI, smoking status, and the prevalence of hypertension, diabetes mellitus, and hypercholesterolemia on CKD (Table VII). Age, diabetes mellitus, hypertension, sex, smoking, F10 genotype (dominant model), AKAP12 genotype (recessive model), MYO7B genotype (dominant model), JPH3 genotype (dominant model), PCSK2 genotype (dominant model), PITRMI genotype (dominant model), and hypercholesterolemia, in descending order of statistical significance, were significant $(\mathrm{P}<0.05)$ and independent determinants of CKD.

\section{Discussion}

We examined the possible relations of 40 gene polymorphisms to the prevalence of CKD in 4,829 Japanese individuals. Our association study with three steps of analysis (chi-square test, multivariable logistic regression analysis, and stepwise forward selection procedure) revealed that the $\mathrm{C} \rightarrow \mathrm{T}$ polymorphism of F10 was significantly (FDR $<0.05)$ associated with CKD in such individuals, with the $T$ allele representing a risk factor for this condition.

Coagulation factor X (F10), the zymogen of a vitamin $\mathrm{K}$-dependent serine protease, plays a pivotal role in the coagulation cascade (22). It can be activated through either the contact-activated (intrinsic) pathway or the tissue-factor (extrinsic) pathway (23). F10 is composed of light and heavy chains held together by a disulfide bond (24) as well as synthesized in the liver as a single-chain molecule (25). Activated F10 catalyzes the conversion of prothrombin to thrombin in the presence of phospholipids and calcium ions (26). Thrombin then converts fibrinogen to insoluble fibrin. F10 deficiency results in a serious bleeding disorder which points to an essential role for F10 in hemostasis (22).

F10 is located at chromosome $13 \mathrm{q} 34$ in close proximity to $F 7$ and spans $\sim 25$ kilobases, containing eight exons (27). The overall domain structure of F10 is highly homologous to that of the other vitamin K-dependent coagulation factors. $F 10$ is primarily expressed in the liver (28). The $-40 \mathrm{C} \rightarrow \mathrm{T}$ polymorphism in the promoter region of $F 10$ was previously associated with coronary heart disease, with the $T$ allele representing a risk factor for this condition (29). However, another study did not detect the relation of this polymorphism to plasma F10 level or to thrombosis risk (30). Although the deleterious influence of the $T$ allele on coronary heart disease cannot be explained by its effect on the circulating level of F10, the previous observation (29) suggested F10 as a candidate susceptibility gene for atherothrombotic disease. We have now shown that the $\mathrm{C} \rightarrow \mathrm{T}$ polymorphism of $\mathrm{F} 10$ was significantly associated with the prevalence of CKD, with the $T$ allele representing a risk factor for CKD. Effects of this polymorphism on the development of atherothrombosis may account for its association with CKD. Given that the $T$ allele frequency of this polymorphism was low, validation of our findings is required in large independent subject panels.

Our results suggest that PITRM1, PCSK2, JPH3, MYO7B, and $A K A P 12$ were also candidate genes for CKD in Japanese individuals. The proprotein convertase 2 gene (PCSK2) encodes the insulin processing protease. The variant of this gene was shown to be associated with type 2 diabetes mellitus in Japanese individuals, with the $A 1$ allele (21 CA repeats) representing a risk factor for this condition (31). Although mutations in certain myosins can cause diseases in humans and mice (32), the myosin-VIIb gene (MYO7B) has not been related to human disease. An antibody to MYO7B labeled proximal tubule cells of the kidney, specifically the distal tips of apical microvilli on these transporting epithelial cells (33). These observations implicate $M Y O 7 B$ as a candidate susceptibility gene for renal dysfunction. The A kinase anchor protein 12 (AKAP12) was originally identified as a cytoplasmic antigen recognized by myasthenia gravis sera (34). The expression of Akap12 was decreased by hypoxia and upregulated by reoxygenation at the transcriptional level in rats (35). We have now shown that the $\mathrm{C} \rightarrow \mathrm{G}$ polymorphism of $P C S K 2$, the $\mathrm{C} \rightarrow \mathrm{T}$ polymorphism of $M Y O 7 B$, and the $\mathrm{A} \rightarrow \mathrm{G}$ polymorphism of $A K A P 12$ were associated with $\mathrm{CKD}$, with the $G, T$, and $G$ alleles, respectively, representing risk factors for this condition, although the underlying mechanisms remain to be determined.

The pitrilysin metallopeptidase 1 gene (PITRMI) is located at chromosome 10p15.2. The mitochondrial PITRM1 functions as a peptide scavenger in the mitochondrial matrix, clearing mitochondria from toxic peptides and protecting them against pathogenic peptide intruders (36). Polymorphisms of PITRM1 have not been related to human disease. JPH3 encodes junctophilin-3, a member of a conserved family of proteins that are components of junctional complexes (37). Holmes et al (38) demonstrated a CAG/CTG repeat expansion of $\geq 40$ triplets in an alternatively spliced exon of $J P H 3$ in affected members of an African American family with Huntington disease-like 2. 
We have now shown that the $\mathrm{C} \rightarrow \mathrm{G}$ polymorphism of PITRMI and the $\mathrm{C} \rightarrow \mathrm{G}$ polymorphism of $\mathrm{JPH} 3$ were associated with CKD, with both $G$ alleles protecting against this condition, although the molecular mechanisms remain unclear.

Our study has several limitations: (i) we used eGFR instead of directly measured GFR to define CKD, (ii) we have not obtained information on the underlying renal disease or the primary cause of CKD in each subject with CKD. Such information could be obtained by detailed clinical examination, including renal biopsy, but such diagnostic procedures are not considered feasible for a study whose subjects are recruited from the general population, (iii) it is possible that one or more of the polymorphisms associated with CKD in the present study are in linkage disequilibrium with other polymorphisms in the same gene or in other nearby genes that are actually responsible for the development of this condition, (iv) the functional relevance of the identified polymorphisms to gene transcription or to protein structure or function was not determined in the present study, (v) although we adopted the criterion of FDR $<0.05$ for association to compensate for the multiple comparisons of genotypes with CKD, it is not possible to exclude completely potential statistical errors such as false positives, (vi) although a previous study (39) showed smoking to be a risk factor for CKD, the frequency of smoking was lower in subjects with CKD than in controls in the present study. Selection bias thus could not be excluded completely in the present study.

In conclusion, our present results suggest that the $\mathrm{C} \rightarrow \mathrm{T}$ polymorphism of F1O is significantly associated with CKD in Japanese individuals. Determination of genotype for this polymorphism may prove informative for assessment of the genetic risk for CKD in such individuals. Validation of our findings will require their replication with independent subject panels as well as long-term follow-up to examine the association of the identified genetic variants with the rate of decline in eGFR.

\section{Acknowledgements}

This work was supported in part by Grants-in-Aid from the Scientific Research from the Ministry of Education, Culture, Sports, Science, and Technology of Japan (nos. 18209023, 18018021, and 19659149 to Y.Y.).

\section{References}

1. Imai E, Matsuo S, Makino H, et al: Chronic Kidney Disease Japan Cohort (CKD-JAC) study: design and methods. Hypertens Res 31: 1101-1107, 2008

2. Weiner DE, Tighiouart H, Amin MG, et al: Chronic kidney disease as a risk factor for cardiovascular disease and all-cause mortality: a pooled analysis of community-based studies. J Am Soc Nephrol 15: 1307-1315, 2004.

3. Jafar TH, Stark PC, Schmid CH, et al: Progression of chronic kidney disease: the role of blood pressure control, proteinuria, and angiotensin-converting enzyme inhibition: a patient-level meta-analysis. Ann Intern Med 139: 244-252, 2003.

4. National Kidney Foundation: K/DOQI clinical practice guidelines for chronic kidney disease: evaluation, classification, and stratification. Am J Kidney Dis 39: S1-S266, 2002.

5. Gharavi AG, Yan Y, Scolari F, et al: IgA nephropathy, the most common cause of glomerulonephritis, is linked to 6q22-23. Nat Genet 26: 354-357, 2000.

6. Hanson RL, Craig DW and Millis MP: Identification of PVT1 as a candidate gene for end-stage renal disease in type 2 diabetes using a pooling-based genome-wide single nucleotide polymorphism association study. Diabetes 56: 975-983, 2007.
7. Yoshida T, Kato K, Fujimaki T, et al: Association of a polymorphism of the apolipoprotein $\mathrm{E}$ gene with chronic kidney disease in Japanese individuals with metabolic syndrome. Genomics 93: 221-226, 2009

8. Yoshida T, Kato K, Yokoi K, et al: Association of genetic variants with chronic kidney disease in Japanese individuals with type 2 diabetes mellitus. Int J Mol Med 23: 529-537, 2009.

9. Doi K, Noiri E, Nakao A, Fujita T, Kobayashi S and Tokunaga K: Functional polymorphisms in the vascular endothelial growth factor gene are associated with development of end-stage renal disease in males. J Am Soc Nephrol 17: 823-830, 2006.

10. Wetmore JB, Hung AM, Lovett DH, Sen S, Quershy O and Johansen KL: Interleukin-1 gene cluster polymorphisms predict risk of ESRD. Kidney Int 68: 278-284, 2005.

11. Matsuo S, Imai E, Horio M, et al: Revised equations for estimated GFR from serum creatinine in Japan. Am J Kidney Dis 53: 982-992, 2009.

12. Go AS, Chertow GM, Fan D, McCulloch CE and Hsu CY: Chronic kidney disease and the risks of death, cardiovascular events, and hospitalization. N Engl J Med 351: 1296-1305, 2004.

13. Imai E, Horio M, Yamagata K, et al: Slower decline of glomerular filtration rate in the Japanese general population: a longitudinal 10-year follow-up study. Hypertens Res 31: 433-441, 2008.

14. Yamada Y, Fuku N, Tanaka M, et al: Identification of CELSR1 as a susceptibility gene for ischemic stroke in Japanese individuals by a genome-wide association study. Atherosclerosis (published online).

15. Ishizaka N, Ishizaka Y, Toda E, et al: Association between chronic kidney disease and carotid intima-media thickening in individuals with hypertension and impaired glucose metabolism. Hypertens Res 30: 1035-1041, 2007.

16. Yoshida T, Kato K, Fujimaki T, et al: Association of genetic variants with chronic kidney disease in Japanese individuals. Clin J Am Soc Nephrol 4: 883-890, 2009.

17. Yoshida T, Kato K, Yokoi K, et al: Association of candidate gene polymorphisms with chronic kidney disease in Japanese individuals with hypertension. Hypertens Res 32: 411-418, 2009.

18. Yoshida T, Kato K, Yokoi K, et al: Association of gene polymorphisms with chronic kidney disease in high- or low-risk subjects defined by conventional risk factors. Int J Mol Med 23: 785-792, 2009.

19. Yoshida T, Kato K, Yokoi K, et al: Association of genetic variants with chronic kidney disease in individuals with different lipid profiles. Int J Mol Med 24: 233-246, 2009.

20. Itoh Y, Mizuki N, Shimada T, et al: High-throughput DNA typing of HLA-A, -B, -C, and -DRB1 loci by a PCR-SSOP-Luminex method in the Japanese population. Immunogenetics 57: 717-729, 2005.

21. Benjamini Y and Hochberg Y: Controlling the false discovery rate: a practical and powerful approach to multiple testing. J Royal Stat Soc Ser B 57: 289-300, 1995.

22. Cooper DN, Millar DS, Wacey A, Pemberton S and Tuddenham EG: Inherited factor $\mathrm{X}$ deficiency: molecular genetics and pathophysiology. Thromb Haemost 78: 161-172, 1997.

23. Millar DS, Elliston L, Deex P, et al: Molecular analysis of the genotype-phenotype relationship in factor X deficiency. Hum Genet 106: 249-257, 2000

24. Di Scipio RG, Hermodson MA, Yates SG, et al: A comparison of human prothrombin, factor IX (Christmas factor), factor X (Stuart factor), and protein S. Biochemistry 16: 698-706, 1977.

25. Graves CB, Munns TW, Willingham AK, et al: Rat factor X is synthesized as a single chain precursor inducible by prothrombin fragments. J Biol Chem 257: 13108-13113, 1982.

26. Mann KG, Nesheim ME, Church WR, Haley $P$ and Krishnaswamy S: Surface-dependent reactions of the vitamin K-dependent enzyme complexes. Blood 76: 1-16, 1990.

27. Leytus SP, Foster DC, Kurachi K and Davie EW: Gene for human factor X: a blood coagulation factor whose gene organization is essentially identical with that of factor IX and protein C. Biochemistry 25: 5098-5102, 1986.

28. Hung HL and High KA: Liver-enriched transcription factor HNF-4 and ubiquitous factor NF-Y are critical for expression of blood coagulation factor X. J Biol Chem 26: 2323-2331, 1996.

29. Schüttrumpf J, Jimenez-Boj E, Graf S, Huber K and Watzke HH: A genetic variant in the promoter of coagulation factor $\mathrm{X}$ increases the risk of acute coronary syndromes. Thromb Haemost (Suppl S): 509, 1999

30. De Visser MC, Poort SR, Vos HL, Rosendaal FR and Bertina RM: Factor $\mathrm{X}$ levels, polymorphisms in the promoter region of factor $\mathrm{X}$, and the risk of venous thrombosis. Thromb Haemost 85: 1011-1017, 2001 
31. Yoshida H, Ohagi S, Sanke T, Furuta H, Furuta M and Nanjo K: Association of the prohormone convertase 2 gene (PCSK2) on chromosome 20 with NIDDM in Japanese subjects. Diabetes 44: 389-393, 1995.

32. Weil D, Levy G, Sahly I, et al: Human myosin VIIA responsible for the Usher 1B syndrome: a predicted membrane-associated motor protein expressed in developing sensory epithelia. Proc Natl Acad Sci USA 93: 3232-3237, 1996.

33. Chen ZY, Hasson T, Zhang DS, et al: Myosin-VIIb, a novel unconventional myosin, is a constituent of microvilli in transporting epithelia. Genomics 72: 285-296, 2001.

34. Gordon T, Grove B, Loftus JC, et al: Molecular cloning and preliminary characterization of a novel cytoplasmic antigen recognized by myasthenia gravis sera. J Clin Invest 90: 992-999, 1992 .
35. Lee SW, Kim WJ, Choi YK, et al: SSeCKS regulates angiogenesis and tight junction formation in blood-brain barrier. Nat Med 9: 900-906, 2003.

36. Falkevall A, Alikhani N, Bhushan S, et al: Degradation of the amyloid beta-protein by the novel mitochondrial peptidasome, PreP. J Biol Chem 281: 29096-29104, 2006.

37. Takeshima H, Komazaki S, Nishi M, et al: Junctophilins: a novel family of junctional membrane complex proteins. Mol Cell 6: 11-22, 2000.

38. Holmes SE, O'Hearn E, Rosenblatt A, et al: A repeat expansion in the gene encoding junctophilin-3 is associated with Huntington disease-like 2. Nat Genet 29: 377-378, 2001.

39. Orth SR: Smoking and the kidney. J Am Soc Nephrol 13: 1663-1672, 2002 